\title{
Expression of estrogen and progesterone receptors in angioleiomyoma of the nasal cavity of six patients
}

\author{
GUOCHEN ZHU ${ }^{1 *}$, DAJIANG XIAO ${ }^{1 *}$ and PING SUN ${ }^{2}$ \\ Departments of ${ }^{1}$ Otolaryngology-Head and Neck Surgery and ${ }^{2}$ Pathology, Wuxi Second People's Hospital, \\ Affiliated to Nanjing Medical University, Wuxi, Jiangsu 214002, P.R. China \\ Received February 13, 2015; Accepted January 18, 2016
}

DOI: $10.3892 / \mathrm{ol} .2016 .4230$

\begin{abstract}
Angioleiomyoma of the nasal cavity is extremely rare. There are only a small number of studies in the literature that demonstrate that the estrogen receptor (ER) and progesterone receptor (PR) are expressed in angioleiomyoma, and the results from these studies are inconsistent. The present study identified 6 patients with nasal angioleiomyoma that were treated between 2004 and 2013. All patients underwent endoscopic surgery and were followed-up for 1-10 years. Resected tumors were investigated for the presence of ER and PR using immunoperoxidase staining. Of the 6 patients, 4 were men and 2 were woman. The mean age of the patients was 60.5 years. The tumors of the 6 patients were identified in the nasal septum, middle turbinate, inferior turbinate, lateral wall of the nasal cavity and nasal vestibule. The clinical manifestations reported by the patients consisted of a painless mass, recurrent epistaxis and nasal obstruction. There were no specific features observed in any of the patients using computed tomography or magnetic resonance imaging. All the patients underwent tumor dissection visualized with a nasal endoscope and recovered without recurrence or malignancy of the tumor post-surgery. Hematoxylin and eosin and immunoperoxidase staining confirmed the diagnosis of angioleiomyoma in all patients. In 5 patients the nuclei of the smooth muscle tumor cells markedly expressed ER and PR. To the best of our knowledge, the present study is the first to demonstrate that ER and PR are clearly expressed in nasal angioleiomyoma. The present study suggests that the sex hormones are possibly associated with the growth of angioleiomyoma.
\end{abstract}

Correspondence to: Dr Guochen Zhu, Department of Otolaryngology-Head and Neck Surgery, Wuxi Second People's Hospital, Affiliated to Nanjing Medical University, 68 Zhongshan Road, Wuxi, Jiangsu 214002, P.R. China

E-mail: zgc2003doctor@sina.com

*Contributed equally

Key words: angioleiomyoma, nasal cavity, estrogen receptor, progesterone receptor, surgical treatment

\section{Introduction}

Angioleiomyoma, or vascular leiomyoma, is an uncommon benign tumor that consists of a mixture of well-differentiated smooth muscle cells and prominent thick-walled vessels. Angioleiomyoma usually occurs in the subcutis of extremities, often developing in lower extremities, and presents as a solitary, small, painful, cutaneous solid-type mass. Angioleiomyoma located in the nasal cavity is uncommon, and studies of nasal angioleiomyoma with $>2$ patients are extremely rare (1-29). To the best of our knowledge, the cases of $~ 50$ patients with nasal angioleiomyoma have been reported in the literature since the first case was reported by Maesaka et al in 1966 (5).

Angioleiomyomas of the nasal cavity typically grow slowly and the majority of these tumors measure $<2 \mathrm{~cm}$ in size (6-29). Patients may remain asymptomatic for a long time, and may present with nasal obstruction, recurrent epistaxis, nasal discharge, facial pain and headache (6-29). The peak incidence of angioleiomyomas of the nasal cavity is between the third and sixth decades of life and the tumor is more common in women (6-29). Angioleiomyoma is rarely diagnosed prior to surgery, as the physical and radiological findings are not tumor-specific in the majority of cases (5-12).

Surgical excision is the recommended form of treatment as has been documented in previous studies. The advent of nasal endoscopes has opened new avenues for surgeons to deal with these tumors. In general, the majority of tumors can be totally excised through the transnasal endoscopic approach, while a smaller number of cases may be removed with craniofacial resection $(2,11,26,29)$.

Hormone receptors, including the estrogen receptor (ER) and progesterone receptor (PR), may be involved in the tumorigenesis of extra-uterine smooth muscle tumors, including uterine angioleiomyoma (6-15,30-34). To date, only a small number of studies have demonstrated that hormone receptors are expressed in angioleiomyoma of the nasal cavity and nasal tip, and there are no studies reporting ER expression (6-12).

Complete excision and pathological examination are essential for a final diagnosis. A possibility of malignant degeneration has been reported. If an angioleiomyoma recurs, it should be treated as a low-grade malignancy (35).

The present study retrospectively reports the clinical manifestations, imaging features, histological characteristics, and management of nasal angioleiomyoma in 6 patients, and 
discusses the importance of ER and PR expression in this tumor.

\section{Patients and methods}

Patients. Subsequent to obtaining approval from the Institutional Review Board of Wuxi Second People's Hospital (Wuxi, Jiangsu, China), the medical records of 6 patients that were diagnosed with angioleiomyoma of the nasal cavity between January 2004 and December 2013 were reviewed. All clinical data are shown in Table I. The data consisted of the age, gender, presentation and duration of symptoms of the patients and clinical features, methods of diagnosis, tumor location, tumor size and subsequent management and surveillance of the patients with angioleiomyoma. Paraffin-embedded sections of surgical specimens from all patients were retrieved and reviewed by a pathologist with 10 years of experience to confirm a diagnosis of angioleiomyoma.

Immunohistochemical staining of tissues. Immunohistochemical staining was performed on $4-\mu \mathrm{m}$-thick formalin-fixed (Xilong Chemical Co., Ltd., Shantou, China) and paraffin-embedded (Shanghai Yiyang Medical Equipment Co., Ltd., Shanghai, China) sections of surgical specimens from 6 patients, according to the manufacturer's protocol of the Elivision ${ }^{\mathrm{TM}}$ plus Polyer HRP (Mouse/Rabbit) IHC kit (catalog no., KIT-9901; Maxim Biotech Inc., Fuzhou, China), which included reagent $\mathrm{A}$ (polymer enhancer) and reagent $\mathrm{B}$ (enzyme-labeled goat anti-mouse/rabbit polymer). All primary antibodies were ready-to-use and purchased from Maxim Biotech Inc., and comprised the following: mouse anti-human calponin monoclonal antibody (catalog no., MAB-0335), mouse anti-human desmin monoclonal antibody (catalog no., MAB-0055), mouse anti-human S-100 monoclonal antibody (catalog no., MAB-0697), mouse anti-human melanoma black-45 monoclonal antibody (catalog no., MAB-0098), mouse anti-human progesterone receptor monoclonal antibody (catalog no., MAB-0675), mouse anti-human estrogen receptor monoclonal antibody (catalog no., MAB-0062), mouse anti-human cluster of differentiation (CD)31 monoclonal antibody (catalog no., MAB-0031), mouse anti-human CD34 monoclonal antibody (catalog no., Kit-0004-2), mouse anti-human Ki-67 monoclonal antibody (catalog no., MAB-0672) and mouse anti-human $\alpha$-smooth muscle actin monoclonal antibody (catalog no., Kit-0006).

Briefly, the slides were deparaffinized with xylene (Xilong Chemical Co., Ltd.) and rehydrated with alcohol (Yang Xu Ditch Chemical Plant, Yixing, China; immersed in 100\% alcohol for 3 min 2 times, 95\% alcohol for 3 min 2 times and $80 \%$ alcohol for $3 \mathrm{~min}$, followed by washing with distilled water for $1 \mathrm{~min}$ ), and antigen retrieval was performed according to the requirements of each primary antibody [CD31, 0.0001M EDTA antigen retrieval solution (pH 9; Maxim Biotech Inc.); $\alpha$-smooth muscle actin does not require antigen retrieval; all other primary antibodies, $0.01 \mathrm{M}$ citrate antigen retrieval solution ( $\mathrm{pH}$ 6; Maxim Biotech Inc.)]; the slides were incubated with 1 drop $(\sim 50 \mu \mathrm{l})$ of primary antibody at $4^{\circ} \mathrm{C}$ overnight, followed by 1 drop $(\sim 50 \mu \mathrm{l})$ of reagent $\mathrm{A}$ for $20 \mathrm{~min}$ and reagent B (secondary antibody; from the Elivision ${ }^{\mathrm{TM}}$ plus Polyer HRP (Mouse/Rabbit) IHC kit) for $30 \mathrm{~min}$ at room temperature.

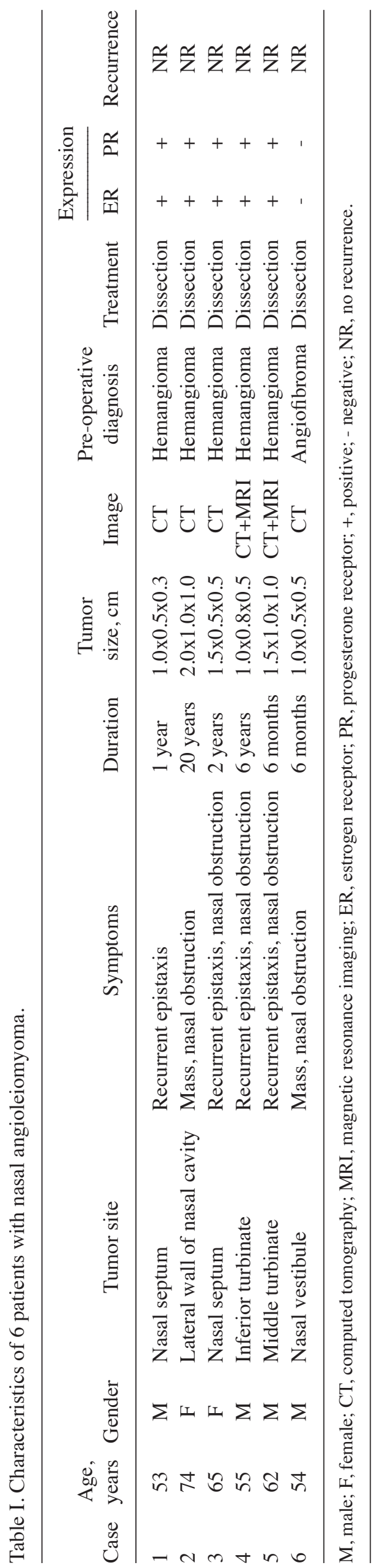





Figure 1. Nasal endoscopic examination revealed that the tumors were located in the (A) nasal vestibule, (B) nasal septum and (C) middle turbinate.
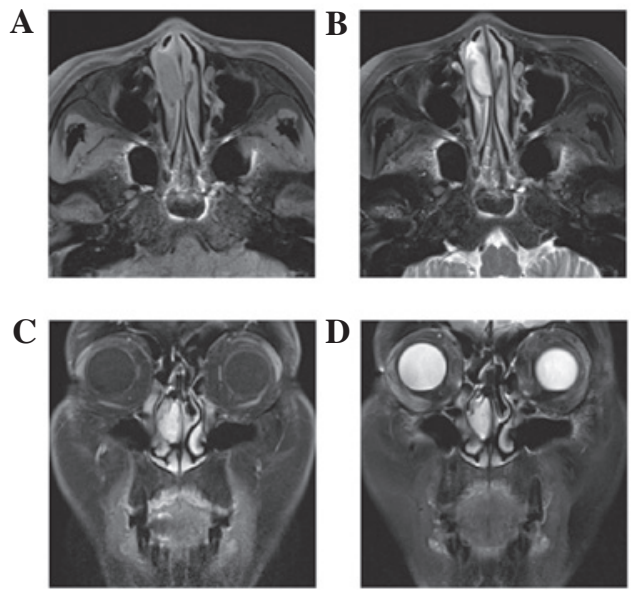

Figure 2. Magnetic resonance imaging of patient 5 demonstrated the presence of a well-defined mass with (A) isointensity to muscle on the T1WI axial scan, (B) heterogeneous hyperintensity on the T2WI axial scan, and clearly delayed enhancement on (C) the contrast T1WI coronal scan and (D) the T2WI coronal scan. T1WI, T1-weighted imaging; T2WI, T2-weighted imaging.

Color reactions were visualized using Ultra Diaminobenzidine (Maxim Biotech Inc.). At the end of each step mentioned above, phosphate buffered saline (Maxim Biotech Inc.) was used to rinse the slides 3 times, for 3 min each time. Finally, the slides were counterstained with hematoxylin and eosin (Maxim Biotech Inc.), washed, dehydrated in alcohol, cleared in xylene, mounted with coverslips (Maxim Biotech Inc.) and visualized using an optical microscope (BX-51, Olympus, Japan).

\section{Results}

Patient characteristics. Of the 6 patients reviewed, 4 were men and 2 were women. All patients were $>50$ years old, and the mean age of the patients was 60.5 years. The primary site of the tumors were the nasal septum, middle turbinate, inferior turbinate, lateral wall of the nasal cavity and nasal vestibule.

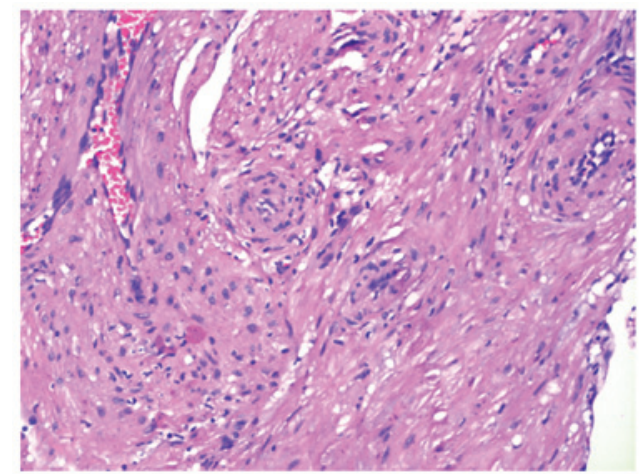

Figure 3. Hematoxylin-eosin staining demonstrated that the tumors were formed of bundles of spindle-shape smooth muscle cells circumscribing numerous slit-like blood vessels. Magnification, x100.
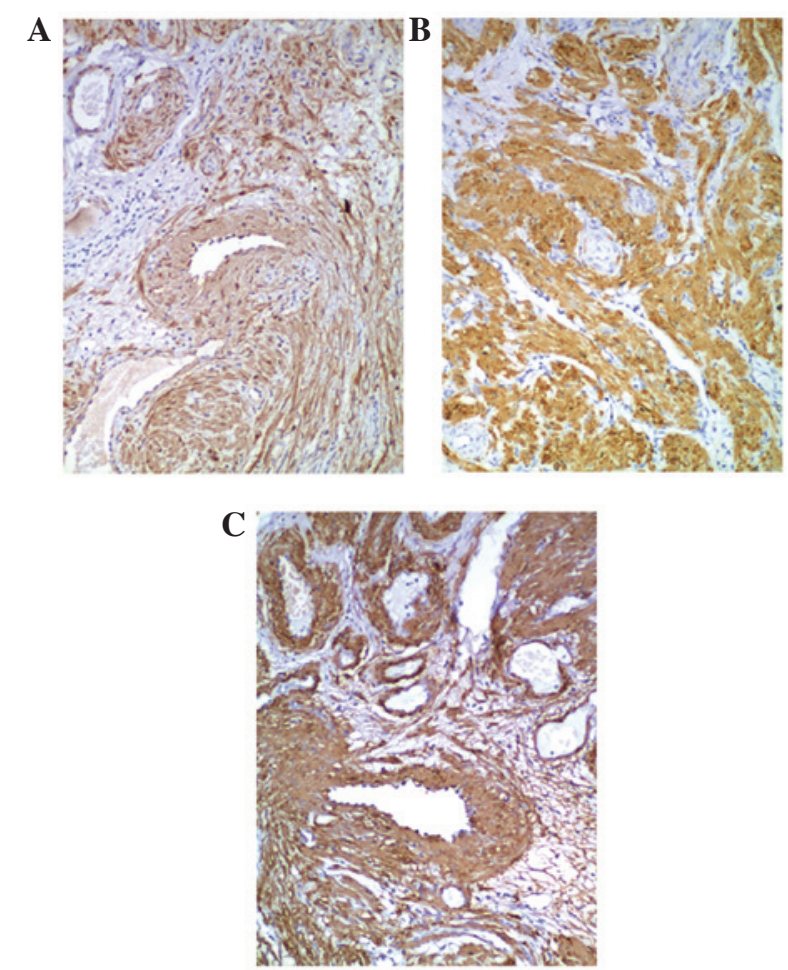

Figure 4. Immunohistochemical staining for (A) calponin, (B) desmin and (C) smooth muscle actin. The cytoplasms of the smooth muscle cells are stained. Magnification, $\mathrm{x} 100$.

The clinical manifestations reported by the patients consisted of a painless mass, recurrent epistaxis and nasal obstruction, and the duration of disease was between 6 months and 20 years.

Diagnosis and treatment. Endoscopic examination revealed that all the tumors were solitary, mobile, well-circumscribed, ovoid or round in shape, with a rough surface. Patient 6 presented with a tumor that exhibited a medium texture, while other patients possessed soft tumors. In total, 4 tumors were dark red, with hemorrhagic areas on the surface, and 2 tumors were bright red, which is a similar color to the nasal mucosa (Fig. 1). All patients underwent a plain, spiral computed tomography (CT) scan. The CT scan revealed solitary nasal lesions with well-defined margins, and the CT 
Table II. Literature review of studies reporting ER and PR expression in various patients with angioleiomyoma.

\begin{tabular}{|c|c|c|c|c|}
\hline \multirow[b]{2}{*}{ First author, year (Ref) } & \multirow[b]{2}{*}{ No. of patients } & \multirow[b]{2}{*}{ Site of tumor } & \multicolumn{2}{|c|}{ Expression } \\
\hline & & & ER & PR \\
\hline Onesti et al, 2012 (6) & 1 & Nasal tip & - & - \\
\hline He et al, 2009 (7) & 1 & Nasal cavity & - & + \\
\hline Kim et al, 2004 (9) & 1 & Nasal cavity & - & - \\
\hline Marioni et al, 2002 (10) & 1 & Nasal cavity & - & + \\
\hline Tseng et al, 2014 (11) & 1 & Nasal cavity & - & + \\
\hline Chen et al, 2007 (12) & 1 & Nasal cavity & - & - \\
\hline Inaba et al, 2015 (30) & 1 & Buccal space & - & + \\
\hline Terada, 2013 (31) & 1 & Lung & - & - \\
\hline Chen et al, 2010 (32) & 1 & Broad ligaments & + & + \\
\hline Hayashi et al, 2009 (33) & 1 & Extra-peritoneal cavity & + & + \\
\hline Di Tommaso et al, 2000 (34) & 10 & Subcutaneous &,$- 100 \%$ &,$+ 60 \%$ \\
\hline
\end{tabular}

ER, estrogen receptor; PR, progesterone receptor; -, negative; +, positive.
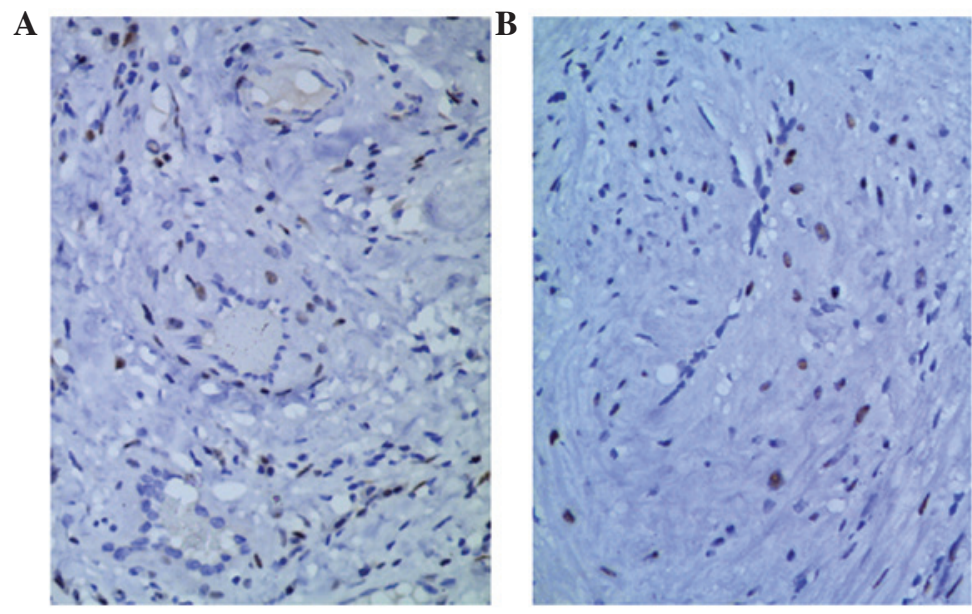

Figure 5. Immunohistochemical staining for (A) estrogen and (B) progesterone receptors. The nuclei of the smooth muscle cells are stained. Magnification, $\mathrm{x} 200$.

values were 46-54 Hounsfield units, which was similar to the CT values of the surrounding non-tumorous soft tissue. No tumors involved the paranasal sinus, orbit or skull base. There were no evident bony erosions observed in any of the patients. Magnetic resonance imaging (MRI) examination was performed in 2 patients, using fast-spin-echo sequence T1-weighted imaging (T1WI) and T2-weighted imaging (T2WI) with fat suppression. The tumor signals in the examination were hypointensive or isointensive to muscle on T1WI, and heterogeneously hyperintensive on T2WI. The tumors exhibited clear delayed enhancement following an intravenous injection of gadolinium-diethylenetriaminepentaacetic acid (Beijing BeiLu Pharmaceutical Co., Ltd., Beijing, China; Fig. 2). Pre-operatively, 5 patients were misdiagnosed with hemangioma and 1 patient was misdiagnosed with angiofibroma.

All patients were treated by tumor resection using a nasal endoscope. Intra-operatively, tumors were easy to completely separate from non-tumorous tissues. The size of the tumors ranged between $1.0 \times 0.5 \times 0.3 \mathrm{~cm}$ and $2.0 \times 1.0 \times 1.0 \mathrm{~cm}$. There was no evident bleeding during surgery in any of the patients. Macroscopically, all lesions had complete capsules and gray-white, brown or dark red sections. All patients recovered without recurrence or malignancy post-operatively, and were followed-up for 1-10 years without evidence of recurrence or malignancy.

Pathological examination. Hematoxylin-eosin staining revealed that the tumors were formed by bundles of spindle-shape smooth muscle cells circumscribing numerous slit-like blood vessels (Fig. 3). The tumors were in close contact with the mucosa, without evidence of ulceration. No mitosis, necrosis or significant nuclear atypia was observed in the tumor cells.

Immunohistochemical staining revealed that the tumor cells exhibited high expression of calponin, desmin and $\alpha$-smooth muscle actin in the cytoplasm of the smooth muscle cells (Fig. 4). Cluster of differentiation (CD)-31 and CD34 were expressed in 
the endothelial cells that lined the vascular spaces. The tumor cells did not express CD31, CD34, human melanoma black-45 or S100. The population of cells expressing Ki-67 was evaluated as a marker for cell proliferation, and the level of positive expression of Ki-67 was $<3 \%$ in all patients. ER and PR were partially expressed in the nuclei of the smooth muscle tumor cells of patients, with the exception of patient 6 (Fig. 5).

\section{Discussion}

The tissue origin of angioleiomyoma is uncertain (2-4); however, hypotheses include angioleiomyomas forming from aberrant undifferentiated mesenchyme and smooth muscle in the wall of blood vessels $(7,8)$. In addition, if the tumor has developed in the nasal vestibule, which is covered with skin, it may have originated from the hair erecting muscles (7). Angioleiomyomas are an extremely rare occurrence in the nasal cavity, which is possibly due to the paucity of smooth muscle in this region (14). Since nasal angioleiomyoma was first identified by Maesaka et al (5) in 1966, this entity has been reported in a small number of papers with 1 or 2 patients. To the best of our knowledge, the current data may be one of the largest groups of patients with nasal angioleiomyoma reported (1-29).

In the 49 cases of nasal angioleiomyoma reported since 1966, the gender of the patients was known in 42 cases and there was a male to female ratio of 5:9; this ratio is similar to that of subcutaneous angioleiomyoma, which has a female predominance (1-29). By contrast, the present results observed a higher male to female ratio. The three most common sites of angioleiomyoma in the nasal cavity are, in order of prevalence, the inferior turbinate, nasal septum and nasal vestibule (1-29). The site of angioleiomyoma development was the superior turbinate and middle turbinate; other sites in the nasal cavity are more rarely observed $(8,21,24)$.

The main clinical symptoms that patients present with are recurrent epistaxis, nasal obstruction and the development of a painless mass, which are non-specific. Occasionally, angioleiomyoma may involve the paranasal sinus, which leads to the destruction of the medial orbit wall (20), a breach of the orbital periosteum (21) and infrequently an extension into the anterior cranial fossa (23). As Figs.1 and 2 reveal, all angioleiomyomas in the present study were confined to the nasal cavity and the tumors were not extremely large in size, and were confirmed by endoscope examination, imaging or surgery.

CT and MRI are the most common imaging methods used for the examination of nasal tumors $(3,36)$, while angiography is only employed in rare cases $(24,37)$. There were no specific features observed during imaging in the present study, but post-contrast images were easily misdiagnosed pre-operatively as hemangioma. However, imaging may aid in determining the extent of tumor invasion and formulating the most appropriate surgical approach, but they may occasionally rule out multi-centric lesions accidentally $(38,39)$.

Sex hormone receptors may play a role in the tumorigenesis of extra-uterine smooth muscle tumors, such as uterine angioleiomyoma. To the best of our knowledge, the presence of the PR and ER have only been evaluated in 20 patients with angioleiomyoma located in various regions of the body, as exhibited in Table II (6,7,9-12,30-34), and these results were inconsistent. Among the 20 patients, 5 patients possessed angioleiomyoma that originated in the nasal cavity, 1 patient possessed angioleiomyoma of the nasal tip, and PR was expressed in 3 patients and ER was not expressed in any of the patients. The present study also evaluated the expression of ER and PR in 6 patients; the two receptors were clearly expressed in 5 patients. To the best of our knowledge, the present study is the first study to demonstrate PR and ER expression in angioleiomyoma of the nasal cavity. The present result is corroborated with ER and PR expression in 2 female patients with angioleiomyoma of the extra-peritoneal cavity and angioleiomyoma of the bilateral broad ligaments, reported by Hayashi et al in 2009 (33) and Chen et al in 2010 (32), respectively. Chen et al (32) suggested that sex hormones may be associated with the growth and degeneration of certain angioleiomyomas, since those receptors are clearly expressed in the nucleus of tumor cells. Additional large-scale studies with more patients are required to elucidate the effect of the PR and ER, particularly the ER, in nasal angioleiomyoma.

Complete surgical excision is the recommended treatment for nasal angioleiomyoma, as reported by previous studies (13,16-29). The surgical approach depends on the size, location and extension of the tumor, and the experience of the surgeon. In the majority of cases, transnasal endoscopic excision is performed successfully, since the majority of the tumors are limited to the nasal cavity, which is detected by radiological examination pre-operatively. When tumors are large or have developed to various locations, a transnasal endoscopic approach is insufficient. Instead, the Caldwell-Luc surgical procedure, local excision, lateral rhinotomy, external ethmoidetomy, medial maxillectomy or craniofacial resection is required (16,20-23). Although angioleiomyomas may be easily detached from the surrounding tissue in the majority of cases, certain studies suggest that a pre-operative endovascular embolism may be an effective method to minimize intra-operative bleeding, particularly in certain lesions with an abundance of vascular structure or a prominent vascular pedicle at the base (24). Singh et al (27) reported that a KTP 532 laser-assisted transnasal endoscopic approach was successfully used in a patient with leiomyoma, to prevent excessive bleeding of the nasal septum. When surgery for intracranial angioleiomyomas is too dangerous, radiotherapy becomes an alternative treatment (40). In the present study, all angioleiomyomas were located in the nasal cavity, so an endoscopic approach with controlled hypotension was sufficient to provide adequate exposure and control over hemostasis without a pre-operative embolism or laser-assisted surgery.

Recurrence and malignant transformation of angioleiomyomas are extremely rare. In 1977, Neviaser et al (41) documented a female patient with angioleiomyoma of the forearm that recurred as leiomyosarcoma 7 years post-operatively. In 1995, Herren et al (42) reported the malignant transformation of angioleiomyoma in the index finger of a 17-year-old patient; the patient relapsed twice and the tumor underwent a malignant alteration. Wide local resection proved to be curative. In 2009, Hayashi et al (33) reported 1 angioleiomyoma that possibly possessed a low malignant potential due to its large size, marked degeneration and abundant expression of mast cells. In 2011, Mahima et al (43) 
reported an unusual angioleiomyoma of the retromolar region, which possessed short-term recurrence and a rapid growth rate.

A differential diagnosis of angioleiomyoma of the nasal cavity may consist of hemangioma, angiomyolipoma, myopericytoma, angiofibroma, leiomyosarcoma and neurofibroma $(7,10,44,45)$. In hemangioma, the intervascular stroma does not have smooth muscle bundles, which are observed in angioleiomyomas. Angiomyolipomas are composed of blood vessels, smooth muscle cells and adipose cells, which express HMB-45. Myopericytomas are formed of thin-walled vascular channels with round or ovoid myopericytes, which express smooth muscle actin (SMA) and focally express, or do not express, desmin. Angiofibromas usually develop in male teenagers and are tumors with proliferated thin-walled vascular channels in a stroma containing round, stellate or spindle-shaped fibroblasts, but without smooth muscle. They express CD34 and do not express SMA. A leiomyosarcoma is a malignant smooth muscle tumor with nuclear atypia and mitosis. A tumor that does not express S100 protein cannot be a neurogenic tumor, such as neurofibroma, neurilemmoma and neuroma, since the S100 protein is only expressed by neurogenic tumors.

In summary, to the best of our knowledge, the present study is the first study to report the expression of the two hormone receptors ER and PR in angioleiomyoma of the nasal cavity. The present study suggests that sex hormones are possibly associated with the growth of angioleiomyoma.

\section{References}

1. Woo KS, Kim SH, Kim HS and Cho PD: Clinical experience with treatment of angioleiomyoma. Arch Plast Surg 41: 374-378, 2014.

2. Yoon TM, Yang HC, Choi YD, Lee DH, Lee JK and Lim SC: Vascular leiomyoma in the head and neck region: 11 years experience in one institution. Clin Exp Otorhinolaryngol 6: 171-175, 2013

3. Liu Y, Li B, Li L, Liu Y, Wang C and Zha L: Angioleiomyomas in the head and neck: A retrospective clinical and immunohistochemical analysis. Oncol Lett 8: 241-247, 2014.

4. Wang CP, Chang YL and Sheen TS: Vascular leiomyoma of the head and neck. Laryngoscope 114: 661-665, 2004.

5. Maesaka A, Keyaki Y and Nakahashi T: Nasal angioleiomyoma and leiomyosarcoma: Report of 2 cases. Otologia (Fukuoka) 12: 42-47, 1966.

6. Onesti MG, Maruccia M, Carella S, Rossi A, Soda G and Scuderi N: Subcutaneous angioleiomyoma of the nasal tip. Report of a rare case. In Vivo 26: 1091-1094, 2012.

7. He J, Zhao LN, Jiang ZN and Zhang SZ: Angioleiomyoma of the nasal cavity: A rare cause of epistaxis. Otolaryngol Head Neck Surg 141: 663-664, 2009

8. Meher R and Varshney S: Leiomyoma of the nose. Singapore Med J 48: e275-e276, 2007.

9. Kim SJ, Hong SH and Roh MS: Angioleiomyoma of the nasal cavity: A case report. Korean J Pathol 38: 181-183, 2004.

10. Marioni G, Marchese-Ragona R, Fernandez S, Bruzon J, Marino F and Staffieri A: Progesterone receptor expression in angioleiomyoma of the nasal cavity. Acta Otolaryngol 122 408-412, 2002

11. Tseng PY,Lai YS, Chen MK and Shen KH: Progesterone receptor expression in sinonasal leiomyoma: A case report and review of the literature. Int J Clin Exp Pathol 7: 1224-1228, 2014.

12. Chen CJ, Lai MT, Chen CY and Fang CL: Vascular leiomyoma of the nasal cavity: Case report. Chin Med J (Engl) 120: 350-352, 2007.

13. Purohit GN, Agarwal N and Agarwal R: Leiomyoma arising from septum of nose. Indian J Otolaryngol Head Neck Surg 63 (Suppl 1): 64-67, 2011.

14. Barr GD, More IA and McCallum HM: Leiomyoma of the nasal septum. J Laryngol Otol 104: 891-893, 1990.

15. Trott MS, Gewirtz A, Lavertu P, Wood BG and Sebek BA: Sinonasal leiomyomas. Otolaryngol Head Neck Surg 111: 660-664, 1994.
16. Schwartzman J and Schwartzman J: Leiomyoangioma of paranasal sinuses: Case report. Laryngoscope 83: 1856-1858, 1973.

17. Wolfowitz BL and Schmaman A: Smooth-muscle tumours of the upper respiratory tract. S Afr Med J 47: 1189-1191, 1973.

18. Ardekian L, Samet N, Talmi YP, Roth Y, Bendet E and Kronenberg J: Vascular leiomyoma of the nasal septum. Otolaryngol Head Neck Surg 114: 798-800, 1996.

19. Hanna GS, Akosa AB and Ali MH: Vascular leiomyoma of the inferior turbinate--report of a case and review of the literature. J Laryngol Otol 102: 1159-1160, 1988.

20. Zijlker TD and Visser R: A vascular leiomyoma of the ethmoid. Report of case. Rhinology 27: 129-135, 1989.

21. Harcourt JP and Gallimore AP: Leiomyoma of the paranasal sinuses. J Laryngol Otol 107: 740-741, 1993.

22. Khan MHZ, Jones AS and Haqqani MT: Angioleiomyoma of the nasal cavity - report of a case and review of the literature. J Laryngol Otol 108: 244-246, 1994.

23. Nicolai P, Redaelli de Zinis LO, Facchetti F, Maroldi R and Antonelli AR: Craniofacial resection for vascular leiomyoma of the nasal cavity. Am J Otolaryngol 17: 340-344, 1996.

24. Nall AV, Stringer SP and Baughman RA: Vascular leiomyoma of the superior turbinate: First reported case. Head Neck 19: 63-67, 1997.

25. Murono S, Ohmura T, Sugimori S and Furukawa M: Vascular leiomyoma with abundant adipose cells of the nasal cavity. Am J Otolaryngol 19: 50-53, 1998.

26. Bloom DC, Finley JC Jr, Broberg TG and Cueva RA: Leiomyoma of the nasal septum. Rhinology 39: 233-235, 2001.

27. Singh R, Hazarika P, Balakrishnan R, Gangwar N and Pujary P: Leiomyoma of the nasal septum. Indian J Cancer 45: 173-175, 2008.

28. Campelo VE, Neves MC, Nakanishi M and Voegels RL: Nasal cavity vascular leiomyoma: Case report and literature review. Rev Bras Otorrinolaringol (Engl Ed) 74: 147-150, 2008.

29. Navarro Júnior CR, Fonseca AS, Mattos JR and Andrade NA: Angioleiomyoma of the nasal septum. Braz J Otorhinolaryngol 76: 675,2010

30. Inaba T, Adachi $\mathrm{M}$ and Yagisita $\mathrm{H}$ : A case of angioleiomyoma in the buccal space. Odontology 103: 109-111, 2015.

31. Terada T: Vascular leiomyoma of the lung arising from pulmonary artery. Int J Clin Exp Pathol 6: 97-99, 2013.

32. Chen X, Zhang X, Zhang S and Lü B: Angioleiomyomas in the bilateral broad ligaments. Int J Gynecol Pathol 29: 39-43, 2010.

33. Hayashi M, Tomita S, Fukasawa I and Inaba N: Large angioleiomyoma, rich of mast cell and sex hormone receptor expression. Arch Gynecol Obstet 279: 193-197, 2009.

34. Di Tommaso L, Scarpellini F, Salvi F, Ragazzini T, Foschini MP and Foschini MP: Progesterone receptor expression in orbital cavernous hemangiomas. Virchows Arch 436: 284-288, 2000.

35. Sawada Y: Angioleiomyoma of the nasal cavity. J Oral Maxillofac Surg 48: 1100-1101, 1990.

36. Gupte C,Butt SH,Tirabosco R and Saifuddin A: Angioleiomyoma: Magnetic resonance imaging features in ten cases. Skeletal Radiol 37: 1003-1009, 2008.

37. Ranjan S and Singh KT: Gingival angioleiomyoma-infrequent lesion of oral cavity at a rare site. J Oral Maxillofac Pathol 18: 107-110, 2014.

38. Ravikumar C, Veerendrakumar M, Hegde T, Nagaraja D, Jayakumar PN and Shankar SK: Basal ganglionic angioleiomyoma. Clin Neurol Neurosurg 98: 253-257, 1996.

39. Shinde SV, Shah AB, Baviskar RB and Deshpande JR: Primary intracranial multicentric angioleiomyomas. Neurol India 60: 115-117, 2012.

40. Sun L, Zhu Y and Wang H: Angioleiomyoma, a rare intracranial tumor: 3 case report and a literature review. World J Surg Oncol 12: 216-222, 2014.

41. Neviaser RJ and Newman W: Dermal angiomyoma of the upper extremity. J Hand Surg Am 2: 271-274, 1977.

42. Herren DB, Zimmermann A and Büchler U: Vascular leiomyoma in an index finger undergoing malignant transformation. J Hand Surg [Br] 20: 484-487, 1995.

43. Mahima VG, Patil K and Srikanth HS: Recurrent oral angioleiomyoma. Contemp Clin Dent 2: 102-105, 2011.

44. Matsuyama A, Hisaoka M and Hashimoto H: Angioleiomyoma: A clinicopathologic and immunohistochemical reappraisal with special reference to the correlation with myopericytoma. Hum Pathol 38: 645-651, 2007.

45. LaBruna A, Reagan B and Papageorge A: Leiomyoma of the maxillary sinus: A diagnostic dilemma. Otolaryngol Head Neck Surg 112: 595-598, 1995. 